\title{
Diretrizes para Autores
}

- Word (.doc ou .docx., ou última versão), Times New Roman 12, espaçamento entre linhas simples, justificado, $3 \mathrm{~cm}$ (esquerda), $2 \mathrm{~cm}$ (direita); $2,5 \mathrm{~cm}$ (topo) $2 \mathrm{~cm}$ (baixo).

- Não mais do que 9.000 palavras

- Primeira pagina - titulo, resumo e abstract (no máximo 120 palavras);

- Texto e referências bibliográficas. Ilustrações (todas denominadas "Figuras" e numeradas sequencialmente) devem ser enviadas eparadamente (jpeg, resolução 600 dpi.no minimo)

- Nas resenhas, considerar como relevante o comentário e a avaliação crítica das referências escolhidas. Estas deverão ser recentes, do ponto de vista de sua publicação. As resenhas devem ter no máximo 1.500 palavras.

- As referências citadas no texto devem incluir o sobrenome do autor, em caixa baixa, o ano de publicação e página. Evitar o uso de expressões latinas no corpo do texto, como ibid., idem e op. cit., com exceção de apud.

- As notas de rodapé devem ser reduzidas ao mínimo necessário e numeradas seqüencialmente.

\section{Informações para envio de trabalhos}

- Os trabalhos deverão ser enviados em dois arquivos separados pelo site :

http://ojs.c3sl.ufpr.br/ojs/index.php/musica/about/submissions\#online Submissions

- Um dos arquivos deverá conter o texto, propriamente dito, mas sem incluir elementos que permitam identificar a sua autoria. Já o segundo arquivo deverá conter os seguintes itens: o nome do autor, título do artigo, résumé (de no máximo 80 palavras), instituição a qual está ligado, endereço para correspondência, fone e e-mail. Para análise do texto, duas cópias serão encaminhadas aos pareceristas.

- Os artigos enviados deverão ser inéditos e não poderão estar ao mesmo tempo submetidos a outras publicações. Para a publicação posterior do texto em outros periódicos, o autor deverá solicitar o consentimento de música em perspectiva.

- Uma revisão gramatical, ortográfica e normativa do texto deverá ser previamente realizada pelo autor antes de submetê-lo ao periódico.

- Os trabalhos não aceitos para publicação não serão devolvidos.

\section{Condições para submissão:}

Como parte do processo de submissão, os autores devem verificar a conformidade da submissão em relação a todos os itens listados a seguir. As submissões que não estiverem de acordo com as normas serão devolvidas aos autores. 
1. A contribuição é original e inédita, e não está sendo avaliada para publicação por outra revista; caso contrário, justificar em "Comentários ao Editor".

2. O texto segue os padrões de estilo e requisitos bibliográficos descritos em Diretrizes para Autores, na seção Sobre a Revista.

3. A identificação de autoria deste trabalho foi removida do arquivo e da opção Propriedades no Word, garantindo desta forma o critério de sigilo da revista, caso submetido para avaliação por pares (ex.: artigos), conforme instruções disponíveis em Asegurando a Avaliação por Pares Cega.

4. Todos os endereços de páginas na Internet (URLs), incluídas no texto (Ex.: http://www.ibict.br) estão ativos e prontos para clicar.

Política de Privacidade : Os nomes e endereços informados nesta revista serão usados exclusivamente para os serviços prestados por esta publicação, não sendo disponibilizados para outras finalidades ou à terceiros.

\section{Rules for Submission}

- Word (.doc ou .docx., or the latest version), Times New Roman 12, simple space between lines, justified, $3 \mathrm{~cm}$ (left), $2 \mathrm{~cm}$ (right); $2,5 \mathrm{~cm}$ (top) $2 \mathrm{~cm}$ (bottom).

- No more than 9.000 words:

- First page - title, abstract (120 words maximum);

- Text and bibliography. Illustrations (figures/tables/examples, etc.) should also be sent in separate archives (.jpeg, $600 \mathrm{dpi}$. minimum)

- Submitted reviews should justify relevance of comments and critics regarding the chosen reference, which should have been recently published; maximum 1.500 words.

- References on the text should include author's first and last name, year of publication and number of page (Author, YEAR, p. X). Avoid using expressions such as ibid., idem and op. cit. (except for apud.)

- Use of footnotes should be restricted to the absolutely indispensable.

\section{Submitting your article}

- Two

separate

archives

via http://ojs.c3sl.ufpr.br/ojs/index.php/musica/about/submissions\#online Submissions

- One of the archives should contain the actual text eliminating everything which could induce author's identification. The second archive should contain: author's name, abstract (maximum 880 words), institution of affiliation, mailing and e-mail addresses, telephone number. Texts will 
then be submitted to blind evaluation by specialists working on the same research domain whose mother language is that of the submitted article.

- Submitted articles should be unpublished and should not be concomitantly submitted for publication. Once published by Musica em Perspectiva, authour's will need to ask for permission in order to have it published somewhere else.

- Texts should be previously reviewed by the author for correct spelling and use of language.

- Please note that articles which are not in accordance with the rules for submission hereby presented will not be accepted.

\section{Check list before submission}

1. Is the article published or is being submitted elsewhere? If this is the case, justify to the editor ; Each case will be considered by the editorial committee;

2. The text is in accordance with the rules for submission;

3. Author's identification was removed from one of the archives, including Word's Property option in order to guarantee blind evaluation (cf. rules for submission).

4. All the electronic addresses (URLs), included (Ex.: http://www.ibict.br) are active and ready to be accessed;

Privacy : Names and addresses sent to Música em Perspectiva will be used exclusively for the submitted article publication's purposes.

\section{Normes de Soumission}

- Word (.doc ou .docx), ou la dernière version, Times New Roman 12, espace interlignes simple, justifié, $3 \mathrm{~cm}$ (gauche), $2 \mathrm{~cm}$ (droite); 2,5 cm (haut); $2 \mathrm{~cm}$ (bas).

- Maximum 9.000 mots:

- Première page : titre, résumé (120 mots maximum); de 3 à 5 mots-clés;

- Texte;

- Références bibliographiques. Illustrations (figures/tables/exemples, etc.) inclues dans le texte, envoyées séparément également (.jpeg, $600 \mathrm{dpi}$. minimum).

- Soumissions de compte rendus doivent justifier l'importance des commentaires et critiques concernant l'œuvre choisie, récemment publiée ; maximum 1.500 mots.

- Références dans le texte doivent faire l'inclusion du prénom et du nom de famille de l'auteur, année de publication et nombre de pages (Auteur, année en majuscules, p. X). Les expressions telles que ibid., idem et op. cit. doivent être évitées, à l'exception de apud. 
- $\quad$ Notes de bas de page : restreintes aux cas absolument indispensables.

\section{Soumission d'article}

- Deux archives doivent être envoyées à travers le site: . http://ojs.c3sl.ufpr.br/ojs/index.php/musica/about/submissions\#online Submissions

- Dans le premier, tous les indices pouvant révéler le nom de l'auteur doivent être éliminés tandis que dans le deuxième il est nécessaire de faire l'inclusion du nom de l'auteur, l'institution d'appartenance, adresse postale, adresse électronique, numéro de téléphone. Tous les textes seront appréciés par des spécialistes - dont la langue maternelle est celle de l'article soumis - travaillant dans le domaine d'étude en question.

- Les articles soumis doivent être inédits et ne peuvent pas être soumis à d'autres publications simultanément. Suite à une publication par Música em Perspectiva, l'auteur devra demander la permission pour publier l'article ailleurs.

- Textes doivent être préalablement révisés par l'auteur, soignant l'orthographe, la rédaction et les normes de publication.

- Les articles qui ne seraient pas en accord avec les normes proposées pour publication ne seront pas acceptés.

\section{Liste à cocher avant soumission}

1. L'article soumis est déjà publié? Une justificative doit être envoyée à l'éditeur; chaque cas sera considéré par le comité éditorial ;

2. Le texte suit les normes de soumission;

3. L'identification d'auteur est éliminée de l'un des archives, cela est obtenu en incluant l'option propriété du Microsoft Word qui préserve l'évaluation aveugle ( $c f$. normes de soumission).

4. Les adresses électroniques (URLs) inclus dans le texte (Ex: http://www.ibict.br) sont actives et accessibles;

Privacité : Les noms et les adresses envoyées à Música em Perspectiva ne seront utilisées que pour soigner les questions relatives à l'article soumis. 